before the Imaging Genetics chapter, and after the fMRI chapter. Hall and Adjamian provide brief reviews of papers that investigated emotions or cognition with pharmacologically related imaging techniques.

Singh's chapter is not the last in this volume and it is certainly not the least. It was a pleasure to read this chapter that begins with necessary discussion of temporal and spatial resolution of the MEG technique, and then provides a technical review of the methodology. While there is some technical material that touches on the physics of the technique, it is sufficiently described in conceptual terms so that the reader can "get their head around" the subject matter. The chapter includes discussion about integrating MEG with EEG, and coregistering MEG with anatomical MRI, and some consideration is given to associating MEG with $\mathrm{MMRI}$.

The chapter authors of Methods in Mind attempt to integrate a collection of neuroscience research methods and ideas within a single volume. They provide good overviews, some more technical and some more conceptual, of their chosen neuroscience techniques. The clinical neuroscientist who is curious about these various techniques and what they offer, the cognitive neuroscientist looking for an introduction to less familiar techniques, and the graduate student will all appreciate this volume. Clinical applications are mentioned, but this is really a book about research methods. As a clinical neuropsychologist, I have to wonder if this book does not predict what we will be doing in the future. If you choose not to put this book on your shelf, then go find it in your library and read some of the chapters. Clinical neuropsychologists may need to better understand and appreciate these neuroscience methods, especially as these techniques become more applicable to clinical work in the future.

\title{
The New Gold Standard
}

DOI: $10.1017 /$ S1355617708080478

Assessment of Malingered Neuropsychological Deficits. Glenn J. Larrabee (Ed.). 2007.

New York: Oxford University Press, 540 pp., \$69.50 (HB)

\section{Reviewed by David S. Bush, Ph.D., ABPP-CN, ABPN, Private Practice, Palm Beach Gardens,} Florida

Developments in the area of malingering detection parallel several of Thomas Kuhn's observations concerning the nonlinear trend of progress in the history of science (Kuhn, 1962). For example, the naive assumption that traditional neuropsychological assessment procedures would straightforwardly generalize to compensation-seeking populations characterized an earlier status quo, a period Kuhn referred to as normal science. The central fallacy of this assumption was the idea that litigants and other claimants did not, as a group, behave differently from those seen in strictly clinical settings. The untenable presumption that astute clinicians were generally capable of forming accurate judgments about the validity of effort on the basis of observation was another received belief that characterized this now archaic era of practice.

Sparked by surprising insights derived from the clinical application of the binomial theorem, the field of neuropsychology entered a new developmental epoch. The rapid expansion of awareness of anomalous data and the discovery of novel facts defined this developmental stage, all of which posed an enormous challenge to preexisting paradigms. Beginning with the early case study approach of Pankratz et al. (1975) and followed by the work of Pankratz et al. (1987) and Hiscock and Hiscock (1989), it became clear that people sometimes perform close to or below chance-level on simple forced-choice measures for reasons not explained by intellectual limitations or neurocognitive impairment. These early studies triggered a transforma- tional period in our field, translating to a conceptual revolution or paradigm shift in the Kuhnian sense (Kuhn, 1962).

As Kuhn (1962) pointed out, a field's textbooks must be rewritten in the aftermath of a scientific revolution. Fortunately, particularly for those on the front lines of forensic neuropsychology practice, Larrabee's Assessment of Malingered Neuropsychological Deficits reflects the collective effort of many of those who have produced much of the most original and significant research in this area. These authorities provide overviews of the entire malingering research infrastructure, including detailed summaries of evidence-based assessment procedures and considerations of future trends in the definition and detection of malingering. The tables alone are worth the price of admission, and the book follows a highly coherent organizational scheme, making it easy to find pertinent facts and figures. The book is an immediately indispensable resource for every neuropsychologist working in or interested in working in the forensic arena.

Chapters 1 and 2, by Larrabee and Larrabee and Berry, respectively, discuss definitions, research designs, base rate issues, and the fundamentals of classification accuracy statistics. Some readers may object to the fact that other authors revisit the topics of research design and classification statistics in subsequent chapters, but others, especially those who are not fully conversant with the sophisticated quantitative procedures upon which much of this research is based, will benefit from some degree of overlap across instructors. 
One of the principle tenets of malingering detection is that proper diagnosis rests on multiple sources of evidence, while minimizing the risk of false-positive errors (i.e., maintaining high specificity). Implicit in this point is the idea that a determination of malingering, like all diagnoses in neuropsychology, is the byproduct of a careful inferential process, which integrates diverse sources of information. Larrabee and Berry's use of the term "malingering tests" in Chapter 2 is unfortunate because it suggests that the test, not the clinician, determines the presence or absence of the condition. This small confusion is, however, entirely forgivable. Covered in all of 25 pages, Chapters 1 and 2 are as clear a presentation of the basics as I have read.

Beginning with Chapter 3, a review of non-forcedchoice effort tests by Boone and Lu, the next several chapters are organized according to domain type. Lest they be unnecessarily neglected in favor of more sophisticated measures, Boone and Lu state that cross-validation data justify the ongoing use of non-forced-choice measures. In Chapter 4, Grote and Hook remind us that effect sizes of forcedchoice tasks generally trump those of non-forced-choice procedures (Vickery et al., 2001). Grote and Hook reiterate that intentionality, the sine qua non of malingering, is difficult to operationalize. However, Chapter 13, Refining Diagnostic Criteria for Malingering by Larrabee, Greiffenstein, Greve, and Bianchini, the most original chapter and one that could have been fittingly subtitled What and How Much We've Learned, provides a compelling operational explanation of intentionality, easily the most novel insight of the entire volume. As application of the Slick criteria (Slick et al., 1999) has enabled the reliable formation of known groups, the outrageously thorny problem of trying to infer intent from single events or behaviors is circumvented by considering multiple, highly improbable events as indicative of intent. This observation sets up recommendations for improving the Slick criteria. Emerging trends in malingering research are discussed; when certain conditions are met, the aggregation of multiple independent indicators has been shown to be associated with virtually perfect specificity (further elaborated by Larrabee, in press).

In Chapter 6, one of the most informative in the book, Greiffenstein takes up the previously obscure topic of sensory and motor test manifestations of malingering. Unawareness of these data is hazardous; anomalous motor findings occur at very high rates among postconcussive claimants. Some of the very best tables in the book appear in Suhr and Barrash's chapter on attention, memory, and psychomotor speed tasks (Chapter 7). The practicing forensic neuropsychologist can make excellent use of the tables summarizing Reliable Digit Span (RDS) studies, as well as data relevant to a determination of noncredible performance on the Auditory Verbal Learning Test (AVLT) and California Verbal Learning Test (CVLT). In Chapter 8, Greve and Bianchini deliver a scholarly review of so-called frontal lobe tests, which could stand on its own as an historical statement. Due to the uniquely disabling nature of executive dysfunction, careful assessment of motivational impairment in the context of executive tests is critically important. The empirical basis for malingering assessment with measures of executive functioning is not robust, however, leading these authors to counsel caution, especially when dealing with serious injuries. In Chapter 9, Berry and Schipper take up the detection of feigned psychiatric symptoms. Beyond the MMPI-2, there is not a great deal of ground to cover here and some of the newer promising measures have not been around long enough to accumulate much support.

Larrabee contributed chapters on the analysis of atypical neuropsychological test patterns (Chapter 5) and the evaluation of exaggerated health and injury symptomatology (Chapter 10). Both chapters expand on the central theme of Larrabee's research; an examination of the empirical foundation of matters directly relevant to the practice of evidencebased forensic neuropsychology. As usual with Larrabee's work, there is always something new and useful. Rounding out the book are chapters on coaching by Suhr and Gunstad (Chapter 11) and noncredible features of the neurological exam by Albers and Schiffer (Chapter 12). In an era when threats to the security of neuropsychological tests have never been greater, neuropsychologists need to know that forcedchoice measures are highly vulnerable to coaching effects. Albers and Schiffer are both neurologists, and there is much to like about their chapter. They state their preference for using the term noncredible to refer to non-neurological illness behavior and exaggerated exam findings, as this does not commit them to opining about underlying motivation. Of course, many forensic neuropsychologists may feel less constrained because they believe they have more to go on. Nevertheless, it would have been nice to learn about the language the other authors use to describe feigned or exaggerated performance because there is so much variability across practitioners and the descriptive language is often tricky for both experienced and novice neuropsychologists.

Albers and Schiffer use a wonderful term-"symptoms of life"- to refer to high base rate, nonspecific symptoms, which are often relied on in the extreme to justify inferences of traumatic brain injury and other neuropsychological conditions. Some readers will puzzle over their claim that the objectivity of neuropsychological tests is no greater than the neurological examination; one assumes they mean that behavior in both contexts is effort-dependent, but the clarity of this statement needed to be sharpened. When commenting on pain in forensic settings, Albers and Schiffer neglected to cite Bianchini et al. (2005), a seminal work on malingered pain-related disability. Anyone somewhat familiar with this literature will note this glaring omission, which, ironically, was published in a medical journal. In contrast to most of the rest of the book, the Albers and Schiffer chapter contains no technical or quantitative data and uses clinical vignettes. As such, this chapter provides something of an intellectual counterpoint and many readers would appreciate it being placed more in the middle of the book instead of toward the end.

It was not that long ago that any earnest neuropsychologist could maintain a single file of articles on malingering. 
The file would not even be that thick, and it would more or less contain all the substantive work in this area and a photocopy of the Rey 15-Item stimulus page to boot. Times have changed, of course. The application of neuropsychology in medical-legal settings has led to the development of a serious science of malingering detection. Larrabee's Assessment of Malingered Neuropsychological Deficits sets the new gold standard in this genre, at least until the second edition of this book is published.

\section{REFERENCES}

Bianchini, K.J., Greve, K.W., \& Glynn, G. (2005). On the diagnosis of malingered pain-related disability: Lesson from cognitive malingering research. Spine Journal, 5, 404-417.

Hiscock, M. \& Hiscock, C.K. (1989). Refining the forced-choice method for the detection of malingering. Journal of Clinical and Experimental Neuropsychology, 11, 967-974.
Kuhn, T.S. (1962). The Structure of Scientific Revolutions. Chicago: University of Chicago Press.

Larrabee, G.J. (in press). Aggregation across multiple indicators improves the detection of malingering: Relationship to likelihood ratios. The Clinical Neuropsychologist.

Pankratz, L., Binder, L.M., \& Wilcox, L. (1987). Evaluation of an exaggerated somatosensory deficit with symptom validity testing. Archives of Neurology, 44, 798.

Pankratz, L., Fausti, A. \& Peed, S. (1975). A forced-choice technique to evaluate deafness in a hysterical or malingering patient. Journal of Consulting and Clinical Psychology, 43, 421-422.

Slick, D.J., Sherman, E.M.S., \& Iverson, G.L. (1999). Diagnostic criteria for malingered neurocognitive dysfunction: Proposed standards for clinical practice and research. The Clinical Neuropsychologist, 13, 545-561.

Vickery, C.E., Berry, D.T.R., Inman, T.H., Harris, M.J., \& Orey, S.A. (2001). Detection of inadequate effort on neuropsychological testing: A meta-analytic review of selected procedures. Archives of Clinical Neuropsychology, 16, 45-73.

\section{A Frontal Lobe Refresher}

DOI: $10.1017 /$ S135561770808048X

The Frontal Lobes: Development, Function and Pathology. Jarl Risberg and Jordan Grafman (Eds.). 2006. New York: Cambridge University Press. 240 pp., \$70.00 (PB)

Reviewed by Joseph R. Sadek, Ph.D., New Mexico Veteran's Administration Health Care System and University of New Mexico Department of Psychiatry, Albuquerque, New Mexico

The Frontal Lobes: Development, Function and Pathology is an edited volume comprised of chapters that are the result of presentations at the annual Advanced Studies Institute sponsored by the International Neuropsychological Society (INS) and by the Vivian Smith Foundation. The eight chapters represent multiple approaches to studying or understanding the frontal lobes, including: evolution and genetics, primate frontal lobe neuroanatomy, cognitive neuroscience models of frontal lobe functioning, microcircuit models based on single neuron recording, developmental models of frontal lobe functioning, case studies of frontal lobe lesion patients, functional neuroimaging of frontal lobe activity, and postmortem neuropathological findings in demented patients. This is a solid but not comprehensive list of approaches to understanding frontal lobe functioning, but the editors clearly state that this volume is not designed to be an exhaustive review. Rather, it is a volume that is better viewed as the state of the art of selected approaches to frontal lobe research as of about 2004, when most of the authors presented a variation of the chapter to the INS Summer Institute. With these qualifications in mind, I can say that the volume is a useful overview that will inform the reader of current and future directions in frontal lobe research.

The chapters are ordered sensibly, starting with evolution to anatomy and ending with a chapter on clinical syndromes, with developmental and theoretical aspects contained in the middle. However, because there was no clear attempt to unify the chapters, the reader can choose where to start in the book without concern that some prerequisite reading of another chapter was necessary to understand the current chapter. As much as this is an advantage for the selective reader, the lack of coherence is the main weakness of this volume. For example, there are two chapters based on primate research, but I could not find reference in either chapter to its partner in the book. Helen Barbas provides an excellent review of the afferent and efferent connections of primary frontal cortex, including a description of anatomically localizable modality specific regions and their systematic variation in laminar connectivity. XiaoJing Wang reviews the concept of microcircuits and their role in working memory. An example of a missed opportunity for integration here is that it would have been very interesting to hear these authors' opinions about what might comprise a complete functional system of visual working memory, including nonfrontal afferents to visual working memory regions and the importance of laminar architecture.

In between the two primate-focused chapters is Jordan Grafman's concise and coherent summary of eight theoretical approaches to frontal lobe functioning. He frames the summary using a "representation" versus "process" comparison of theories of frontal lobe functioning. Simplistically speaking, "representationalists" believe that the frontal lobe stores representations (especially cognitive represen- 